\title{
ON SECOND ORDER NONCONVEX SWEEPING PROCESS WITH NONCONVEX PERTURBATION
}

\author{
Myelkebir Aitalioubrahim
}

\begin{abstract}
This paper deals with the existence result of solutions of a second order functional differential inclusion, governed by a class of nonconvex sweeping process, with a nonconvex perturbation.
\end{abstract}

\section{Introduction}

Let $H$ be a real separable Hilbert space with the norm $\|\cdot\|$ and the scalar product $\langle\cdot, \cdot\rangle$. For $I$ a segment in $\mathbb{R}$, we denote by $\mathcal{C}(I, H)$ the Banach space of continuous functions from $I$ to $H$ equipped with the norm $\|x(\cdot)\|_{\infty}:=$ $\sup \{\|x(t)\|: t \in I\}$. For $a$ a positive number, we put $\mathcal{C}_{a}:=\mathcal{C}([-a, 0], H)$ and for any $t \in[0, T], T>0$, we define the operator $T(t)$ from $\mathcal{C}([-a, T], H)$ to $\mathcal{C}_{a}$ with $(T(t)(x(\cdot)))(s):=(T(t) x)(s):=x(t+s), s \in[-a, 0]$. For $x \in H$ and $r>0$ let $B(x, r):=\{y \in H:\|y-x\|<r\}$ be the open ball centered at $x$ with radius $r$ and $\bar{B}(x, r)$ be its closure and put $B=B(0,1)$. For $\varphi \in \mathcal{C}_{a}$ and $r>0$ let $B_{a}(\varphi, r):=\{\psi \in H:\|\psi-\varphi\|<r\}$ be the open ball centered at $\varphi$ with radius $r$ and $\bar{B}_{a}(\varphi, r)$ be its closure. For $x \in H$ and for nonempty subsets $A, B$ of $H$ we denote $d_{A}(x)$ or $d(x, A)$ the real inf $\{\|y-x\|: y \in A\}$, $e(A, B):=\sup \left\{d_{B}(x): x \in A\right\}$ and $H(A, B)=\max \{e(A, B), e(B, A)\}$. A multifunction is said to be measurable if its graph is measurable. For more details on measurability theory, we refer the reader to book of Castaing-Valadier [11].

In this paper, we study the existence of solutions of the following nonconvex differential inclusions

$$
\left\{\begin{array}{l}
\ddot{x}(t) \in-N_{C(x(t))}^{p}(\dot{x}(t))+F(t, T(t) x, T(t) \dot{x}) \quad \text { a.e. on }[0, T] \\
\dot{x}(t)=\bar{\varphi}(t) \quad \forall t \in[-a, 0] \\
\dot{x}(t) \in C(x(t)) \quad \forall t \in[0, T] \\
x(t)=\varphi(t) \quad \forall t \in[-a, 0]
\end{array}\right.
$$

Received March 16, 2010; Revised February 6, 2011.

2010 Mathematics Subject Classification. 49K21, 49J21.

Key words and phrases. nonconvex sweeping process, functional differential inclusion, uniformly $\rho$-prox regular sets. 
where $C$ is a set-valued mapping, $N_{C(x(t))}^{p}(\dot{x}(t))$ denotes a prescribed normal cone to the set $C(x(t))$ at $\dot{x}(t), F$ is a set-valued mapping, measurable with respect to the first argument and Lipschitz continuous with respect to the third argument, $\varphi$ and $\bar{\varphi}$ are two continuous functions.

Convex sweeping process was introduced and studied by Moreau (see for example [19]). We refer to [18] and [15] for a complete bibliography on the subject. Note that, the sweeping process is related to the modelization of elasto-plastic materials (see for example [20, 21]). For the sweeping process without convexity, we refer the reader to $[1,4]$ and the references therein.

The second order sweeping process has been studied by several authors. Castaing [9] studied, for the first time, the particular type of second order differential inclusions, $\ddot{x}(t) \in-N_{C(x(t))}(\dot{x}(t)), \dot{x}(t) \in C(x(t))$, where $C$ is a convex compact set-valued mapping. Bounkhel and Laouir-Azzam [6] studied, in finite dimensional space, the perturbed problem $\ddot{x}(t) \in-N_{C(x(t))}(\dot{x}(t))+F(t, \dot{x}(t))$ when $C$ is nonconvex and the multifunction $F$ is bounded with convex values. The same authors proved existence results, in Hilbert space, for the following perturbed problem $\ddot{x}(t) \in-N_{C(x(t))}(\dot{x}(t))+F(t, x(t))$ when $C$ is nonconvex and $F$ is nonconvex and continuous. Bounkhel [5] proved several existence theorems, in separable Hilbert space, for the following general problem, which covers all the problems studied before and mentioned above, $\ddot{x}(t) \in-N_{C(x(t))}^{p}(\dot{x}(t))+F(t, x(t), \dot{x}(t))+G(t, x(t), \dot{x}(t))$, where $C$ is nonconvex such that $C(x)$ is contained in compact convex set or $C$ is anti-monotone and $C(x) \subset l B(l \in \mathbb{R}), F$ is a scalarly upper semicontinuous convex compact set-valued mapping, and $G$ is a nonconvex compact continuous set-valued mapping. Azzam-Laouir [3] proved, in finite dimensional, the existence of solutions of the following problem $\ddot{x}(t) \in-N_{C(x(t))}(\dot{x}(t))+F(t, x(t), \dot{x}(t))$ when $F$ is mixed semicontinuous, $C$ is Lipschiz and $C(x)$ is uniformly $\rho$-prox-regular. Bounkhel and Yarou [8] studied the second order sweeping process with delay $\ddot{x}(t) \in-N_{C(x(t))}(\dot{x}(t))+F(t, T(t) x, T(t) \dot{x})$ when $C$ is Lipschiz, $C(x)$ is uniformly $\rho$-prox-regular, $C(x)$ is contained in compact convex set, and $F$ is scalarily upper semicontinuous with convex weakly compact values.

In this paper, our main purpose is to obtain the existence of solutions of the general problem (1.1), in the case when the perturbation $F$ is a measurable multifunction with respect to the first argument and Lipschitz continuous with respect to the third argument with closed values, and $C$ is Lipschiz multifunction such that $C(x)$ is uniformly $\rho$-prox-regular and $C(x)$ is contained in compact set. Note that the hypotheses imposed on the right-hand side, and methods of the proof are different from the above cited works. Indeed, in this paper

- $F$ is not continuous (contrary to $[5]$ ), it is nonconvex (contrary to $[6,8]$ ) and it is noncompact (contrary to $[5,8]$ ).

- $C$ is nonconvex and not contained in convex set (contrary to $[5,8,9]$ ).

- The space of states is infinite-dimensional (contrary to $[3,6]$ ). 
Furthermore, the construction of the sequence of approximate solutions is different from that used in $[3,5,6,8,9]$.

The paper is organized as follows. In Section 2, we recall some preliminary facts that we need in the sequel while in Section 3, we prove our main result.

\section{Preliminaries and statement of the main result}

We need first to recall some notations and definitions that will be used in all the paper.

Let $V: H \rightarrow \mathbb{R} \cup\{+\infty\}$ be a lower semicontinuous function and $x$ be any point where $V$ is finite. The proximal subdifferential $\partial^{p} V(x)$ of $V$ at $x$ is the set of all $y \in H$, for which there exist $\delta, \sigma>0$ such that for all $x^{\prime} \in x+\delta \bar{B}$

$$
\left\langle y, x^{\prime}-x\right\rangle \leq V\left(x^{\prime}\right)-V(x)+\sigma\left\|x^{\prime}-x\right\|^{2} .
$$

Let $S$ be a nonempty closed subset of $H$ and $x$ be a point in $S$. We recall (see [13]) that the proximal normal cone of $S$ at $x$ is defined by $N_{S}^{p}(x):=\partial^{p} \psi_{S}(x)$, where $\psi_{S}(\cdot)$ denotes the indicator function of $S$, i.e., $\psi_{S}(x)=0$ if $x \in S$ and $+\infty$ otherwise.

Recall now that for a given $\rho \in] 0,+\infty]$, a subset $S$ is uniformly $\rho$-proxregular (see [22]), or equivalently $\rho$-proximally smooth (see [13]), if and only if every nonzero proximal normal to $S$ can be realized by an $\rho$-ball, this means that for all $\bar{x} \in S$ and all $\xi \in N_{S}^{p}(\bar{x}) \backslash\{0\}$ one has

$$
\left\langle\frac{\xi}{\|\xi\|}, x-\bar{x}\right\rangle \leq \frac{1}{2 \rho}\|x-\bar{x}\|^{2}
$$

for all $x \in S$. We make the convention $\frac{1}{\rho}=0$ for $\rho=+\infty$. Recall that for $\rho=+\infty$ the uniform $\rho$-prox-regularity of $S$ is equivalent to the convexity of $S$.

The following propositions summarize some important consequences of uniform prox-regularity needed in the sequel.

Porposition 2.1 ([22]). Let $S$ be a nonempty closed subset in $H$ and $x \in S$. The following assertions hold:

(i) $\partial^{p} d(x, S)=N_{S}^{p}(x) \cap \bar{B}$;

(ii) Let $\rho \in] 0,+\infty]$. If $S$ is uniformly $\rho$-prox-regular, then for all $x \in H$ with $d(x, S)<\rho$ one has $\operatorname{Proj}_{S}(x) \neq \emptyset$ and $\partial^{P} d(x, S)=\partial^{C} d(x, S)$, where $\partial^{C} d(x, S)$ is the Clarke subdifferential of $d(\cdot, S)$ at $x$. So, in such a case, the subdiferential $\partial d(x, S):=\partial^{P} d(x, S)=\partial^{C} d(x, S)$ is a closed convex set in $H$.

(iii) If $S$ is uniformly $\rho$-prox-regular, then for all $x_{i} \in S$ and all $v_{i} \in N_{S}^{p}\left(x_{i}\right)$ with $\left\|v_{i}\right\| \leq \rho(i=1,2)$ one has

$$
\left\langle v_{1}-v_{2}, x_{1}-x_{2}\right\rangle \geq-\left\|x_{1}-x_{2}\right\|^{2} .
$$

As a consequence of (iii) we get that for uniformly $\rho$-prox-regular sets, the proximal normal cone to $S$ coincides with all the normal cones contained in the 
Clarke normal cone at all points $x \in S$, i.e., $N_{S}^{P}(x)=N_{S}^{C}(x)$. In such a case, we put $N_{S}(x):=N_{S}^{P}(x)=N_{S}^{C}(x)$.

Porposition $2.2([7])$. Let $\rho \in] 0,+\infty]$ and $\Omega$ be an open subset in $H$ and let $C: \Omega \rightarrow 2^{H}$ be a Hausdorff-continuous set-valued mapping. Asssume that $C$ has uniformly $\rho$-prox-regular values. Then, the set-valued mapping given by $(z, x) \rightarrow \partial d_{C(z)}(x)$ from $\Omega \times H$ (endowed with the strong topology) to $H$ (endowed with the weak topology) is upper semicontinuous, which is equivalent to the upper semicontinuous of the function $(z, x) \rightarrow \sigma\left(\partial d_{C(z)}(x), p\right)$ for any $p \in$ $H$. Here $\sigma(S, p)$ denotes the support function associated with $S$, i.e., $\sigma(S, p)=$ $\sup _{s \in S}\langle s, p\rangle$.

Let us recall the following lemmas that will be used in the sequel.

Lemma $2.3([23])$. Let $\Omega$ be a nonempty set in $H$. Assume that $F:[a, b] \times \Omega \rightarrow$ $2^{H}$ is a multifunction with nonempty closed values satisfying:

- For every $x \in \Omega, F(\cdot, x)$ is measurable on $[a, b]$;

- For every $t \in[a, b], F(t, \cdot)$ is (Hausdorff) continuous on $\Omega$.

Then for any measurable function $x(\cdot):[a, b] \rightarrow \Omega$, the multifunction $F(\cdot, x(\cdot))$ is measurable on $[a, b]$.

Lemma 2.4 ([23]). Let $G:[a, b] \rightarrow 2^{H}$ be a measurable multifunction and $y(\cdot):[a, b] \rightarrow H$ a measurable function. Then for any positive measurable function $r(\cdot):[a, b] \rightarrow \mathbb{R}^{+}$, there exists a measurable selection $g(\cdot)$ of $G$ such that for almost all $t \in[a, b]$

$$
\|g(t)-y(t)\| \leq d(y(t), G(t))+r(t) .
$$

If $B$ is a bounded set of $H$, then the Kuratowski's measure of noncompactness of $B, \beta(B)$, is defined by

$$
\begin{gathered}
\beta(B)=\inf \{d>0: B \text { admits a finite number of sets with diameter } \\
\text { less than } d\} .
\end{gathered}
$$

In the following lemma we recall some useful properties for the measure of noncompactness $\beta$. For instance see Proposition 9.1 [14].

Lemma 2.5. Let $X$ be an infinite dimensional real Banach space and $D_{1}, D_{2}$ be two bounded subsets of $X$.

(i) $\beta\left(D_{1}\right)=0 \Leftrightarrow D_{1}$ is relatively compact.

(ii) $\beta\left(\lambda D_{1}\right)=|\lambda| \beta\left(D_{1}\right) ; \lambda \in \mathbb{R}$.

(iii) $D_{1} \subseteq D_{2} \Rightarrow \beta\left(D_{1}\right) \leq \beta\left(D_{2}\right)$.

(iv) $\beta\left(D_{1}+D_{2}\right) \leq \beta\left(D_{1}\right)+\beta\left(D_{2}\right)$.

(v) If $x_{0} \in X$ and $r$ is a positive real number, then $\beta\left(B\left(x_{0}, r\right)\right)=2 r$.

Assume that the following hypotheses hold:

(H1) $C: H \rightarrow 2^{H}$ is a $k$-Lipschiz set-valued map with nonempty closed values satisfying 
(a) For each $x \in H, C(x)$ is $\rho$-prox-regular for some fixed $\rho \in] 0,+\infty]$;

(b) There exists a compact subset $L$ of $H$ such that $C(x) \subseteq L$ for all $x \in H$

(H2) $F:[0, b] \times \mathcal{C}_{a} \times \mathcal{C}_{a} \rightarrow 2^{H}$ is a set-valued map with nonempty closed values satisfying

(i) For each $\psi, \phi \in \mathcal{C}_{a}, t \mapsto F(t, \psi, \phi)$ is measurable;

(ii) There exists a function $m(\cdot) \in L^{1}\left([0, b], \mathbb{R}^{+}\right)$such that for all $t \in$ $[0, b]$ and for all $\psi, \phi_{1}, \phi_{2} \in \mathcal{C}_{a}$

$$
H\left(F\left(t, \psi, \phi_{1}\right), F\left(t, \psi, \phi_{2}\right)\right) \leq m(t)\left\|\phi_{1}-\phi_{2}\right\|_{\infty} ;
$$

(iii) For all bounded subset $S$ of $\mathcal{C}_{a} \times \mathcal{C}_{a}$, there exist three functions $g_{S}(\cdot), p_{S}(\cdot), q_{S}(\cdot) \in L^{1}\left([0, b], \mathbb{R}^{+}\right)$such that for all $t \in[0, b]$ and for all $(\psi, \phi) \in S$

$$
F(t, \psi, \phi) \cap \bar{B}\left(0, g_{S}(t)+p_{S}(t)\|\psi\|_{\infty}+q_{S}(t)\|\phi\|_{\infty}\right) \neq \emptyset .
$$

We established the following result:

Theorem 2.6. If assumptions (H1) and (H2) are satisfied, then for all $\varphi, \bar{\varphi} \in$ $\mathcal{C}_{a}$ such that $\bar{\varphi}(0) \in C(\varphi(0))$, there exist $T>0, r>0, M>0$, and a continuous function $x(\cdot):[-a, T] \rightarrow H$ such that $x(\cdot)$ and $\dot{x}(\cdot)$ are absolutely continuous on $[0, T], \dot{x}(\cdot)$ is continuous on $[-a, T]$, and $x(\cdot)$ is a solution of

$$
\left\{\begin{array}{l}
\ddot{x}(t) \in-N_{C(x(t))}^{p}(\dot{x}(t))+F(t, T(t) x, T(t) \dot{x}) \quad \text { a.e. on }[0, T] \\
\dot{x}(t)=\bar{\varphi}(t), \quad \forall t \in[-a, 0] \\
\dot{x}(t) \in C(x(t)), \quad \forall t \in[0, T] \\
x(t)=\varphi(t), \quad \forall t \in[-a, 0]
\end{array}\right.
$$

and satisfies

$$
\|\dot{x}(t)\| \leq k M+2 g(t)+2 p(t)\left(\|\varphi\|_{\infty}+r\right)+2 q(t)\left(\|\bar{\varphi}\|_{\infty}+r\right)
$$

for almost all $t \in[0, T]$.

\section{Proof of the main result}

Fix $\varphi, \bar{\varphi} \in \mathcal{C}_{a}$ such that $\bar{\varphi}(0) \in C(\varphi(0))$. Let $r>0$ and $g(\cdot), p(\cdot), q(\cdot) \in$ $L^{1}\left([0, b], \mathbb{R}^{+}\right)$such that for all $(t, \psi, \phi) \in[0, b] \times \bar{B}_{a}(\varphi, r) \times \bar{B}_{a}(\bar{\varphi}, r)$

$$
F(t, \psi, \phi) \cap \bar{B}\left(0, g(t)+p(t)\|\psi\|_{\infty}+q(t)\|\phi\|_{\infty}\right) \neq \emptyset .
$$

The set $L$ is compact in $H$ and so there exists $M>0$ such that $L \subset M \bar{B}$. For simplification, set $\delta(t)=g(t)+p(t)\left(\|\varphi\|_{\infty}+r\right)+q(t)\left(\|\bar{\varphi}\|_{\infty}+r\right)$ for all $t \in[0, b]$. Let $T_{1}>0$ such that

$$
\int_{0}^{T_{1}}(k M+2 \delta(s)) d s<\inf \left\{\frac{r}{2}, \frac{\rho}{2}\right\} .
$$


For $\varepsilon>0$ set

$$
\begin{aligned}
= & \sup \{\gamma \in] 0, \varepsilon]:\left|\int_{t_{1}}^{t_{2}}(k M+2 \delta(s)) d s\right|<\varepsilon, \\
& \left.\left\|\varphi\left(t_{1}\right)-\varphi\left(t_{2}\right)\right\|<\varepsilon \text { and }\left\|\bar{\varphi}\left(t_{1}\right)-\bar{\varphi}\left(t_{2}\right)\right\|<\varepsilon \text { if }\left|t_{1}-t_{2}\right|<\gamma\right\} .
\end{aligned}
$$

Put

$$
T=\min \left\{T_{1}, \frac{1}{2} \eta\left(\frac{r}{2}\right), b, \frac{r}{2 M}\right\} .
$$

We will used the following lemma to prove main result.

Lemma 3.1. If assumptions ( $\mathrm{H} 1)$ and $(\mathrm{H} 2)$ are satisfied, then for all $n \in \mathbb{N}^{*}$ and for all $y(\cdot) \in L^{1}([0, T], H)$, there exist two continuous mappings $x_{n}(\cdot)$ : $[-a, T] \rightarrow H, u_{n}(\cdot):[-a, T] \rightarrow H$, a step functions $\theta_{n}(\cdot), \bar{\theta}_{n}(\cdot):[0, T] \rightarrow[0, T]$ and $f_{n}(\cdot) \in L^{1}([0, T], H)$ such that

(a) $f_{n}(t) \in F\left(t, T\left(\theta_{n}(t)\right) x_{n}, T\left(\theta_{n}(t)\right) u_{n}\right) \cap \bar{B}\left(0, g(t)+p(t)\left\|T\left(\theta_{n}(t)\right) x_{n}\right\|_{\infty}+\right.$ $\left.q(t)\left\|T\left(\theta_{n}(t)\right) u_{n}\right\|_{\infty}\right), u_{n}\left(\bar{\theta}_{n}(t)\right) \in C\left(x_{n}\left(\bar{\theta}_{n}(t)\right)\right)$ for all $t \in[0, T]$

(b) $\left\|f_{n}(t)-y(t)\right\| \leq d\left(y(t), F\left(t, T\left(\theta_{n}(t)\right) x_{n}, T\left(\theta_{n}(t)\right) u_{n}\right)\right)+\frac{1}{n}$ for all $t \in$ $[0, T]$

(c) $\left(\dot{u}_{n}(t)-f_{n}(t)\right) \in-N_{C\left(x_{n}\left(\bar{\theta}_{n}(t)\right)\right)}\left(u_{n}\left(\bar{\theta}_{n}(t)\right)\right)$ for almost all $t \in[0, T]$;

(d) $\left\|\dot{u}_{n}(t)-f_{n}(t)\right\| \leq k M+\delta(t)$ for almost every $t \in[0, T]$.

Proof. Fix $n \in \mathbb{N}^{*}$ and let $y(\cdot):[0, T] \rightarrow H$ be a measurable function. Consider a sequence $\left(P_{n}\right)_{n}$ of subdivisions of $[0, T]$ :

$$
P_{n}=\left\{0=t_{0}^{n}<t_{1}^{n}<\cdots<t_{i}^{n}<\cdots<t_{2^{n}}^{n}=T\right\},
$$

where $t_{i}^{n}=i \frac{T}{2^{n}}, 0<i<2^{n}$. Let us define the sequences $\left(x_{n}\right)_{n}$ and $\left(u_{n}\right)_{n}$ of approximate solutions as follows. Set $x_{n}(s)=\varphi(s)$ and $u_{n}(s)=\bar{\varphi}(s)$ for all $s \in[-a, 0]$. Put $x_{0}^{n}=\varphi(0)$ and $u_{0}^{n}=\bar{\varphi}(0) \in C\left(x_{n}\left(t_{0}^{n}\right)\right)$. By Lemma 2.3, $t \mapsto F\left(t, T(0) x_{n}, T(0) u_{n}\right)$ is a measurable multifunction, then by Theorem III.40 and Theorem III.41 in [11]

$$
t \mapsto F\left(t, T(0) x_{n}, T(0) u_{n}\right) \cap \bar{B}\left(0, g(t)+p(t)\left\|T(0) x_{n}\right\|_{\infty}+q(t)\left\|T(0) u_{n}\right\|_{\infty}\right)
$$

is a measurable multifunction. In view of Lemma 2.4, there exists a function $f_{0}^{n} \in L^{1}\left(\left[t_{0}^{n}, t_{1}^{n}\right], H\right)$ such that

$$
f_{0}^{n}(t) \in F\left(t, T(0) x_{n}, T(0) u_{n}\right) \cap \bar{B}\left(0, g(t)+p(t)\left\|T(0) x_{n}\right\|_{\infty}+q(t)\left\|T(0) u_{n}\right\|_{\infty}\right)
$$

and

$$
\left\|f_{0}^{n}(t)-y(t)\right\| \leq d\left(y(t), F\left(t, T(0) x_{n}, T(0) u_{n}\right)\right)+\frac{1}{n}
$$

for all $t \in\left[t_{0}^{n}, t_{1}^{n}\right]$. Set

$$
x_{n}(t)=x_{0}^{n}+\left(t-t_{0}^{n}\right) u_{0}^{n}, \quad \forall t \in\left[t_{0}^{n}, t_{1}^{n}\right]
$$


and put $x_{n}\left(t_{1}^{n}\right)=x_{1}^{n}$. By (H1) and (3.1), we have

$$
\begin{aligned}
& d_{C\left(x_{n}\left(t_{1}^{n}\right)\right)}\left(u_{0}^{n}+\int_{t_{0}^{n}}^{t_{1}^{n}} f_{0}^{n}(s) d s\right) \\
\leq & H\left(C\left(x_{n}\left(t_{1}^{n}\right)\right), C\left(x_{n}\left(t_{0}^{n}\right)\right)\right)+\int_{t_{0}^{n}}^{t_{1}^{n}}\left\|f_{0}^{n}(s)\right\| d s \\
\leq & k\left\|x_{n}\left(t_{1}^{n}\right)-x_{n}\left(t_{0}^{n}\right)\right\|+\int_{t_{0}^{n}}^{t_{1}^{n}}\left\|f_{0}^{n}(s)\right\| d s \\
\leq & k \int_{t_{0}^{n}}^{t_{1}^{n}}\left\|u_{0}^{n}\right\| d s+\int_{t_{0}^{n}}^{t_{1}^{n}}\left\|f_{0}^{n}(s)\right\| d s \\
\leq & \int_{t_{0}^{n}}^{t_{1}^{n}}\left(k M+g(s)+p(s)\|\varphi\|_{\infty}+q(s)\|\bar{\varphi}\|_{\infty}\right) d s \\
\leq & \frac{\rho}{2} .
\end{aligned}
$$

As $C$ has uniformly $\rho$-prox-regular values, by Proposition 2.1, we have

$$
\operatorname{Proj}_{C\left(x_{n}\left(t_{1}^{n}\right)\right)}\left(u_{0}^{n}+\int_{t_{0}^{n}}^{t_{1}^{n}} f_{0}^{n}(s) d s\right) \neq \emptyset .
$$

Then, one can choose a point $u_{1}^{n}$ in

$$
\operatorname{Proj}_{C\left(x_{n}\left(t_{1}^{n}\right)\right)}\left(u_{0}^{n}+\int_{t_{0}^{n}}^{t_{1}^{n}} f_{0}^{n}(s) d s\right) .
$$

Note that $u_{1}^{n} \in C\left(x_{n}\left(t_{1}^{n}\right)\right)$ and

$$
\begin{aligned}
& \left\|u_{1}^{n}-\left(u_{0}^{n}+\int_{t_{0}^{n}}^{t_{1}^{n}} f_{0}^{n}(s) d s\right)\right\| \\
= & d_{C\left(x_{n}\left(t_{1}^{n}\right)\right)}\left(u_{0}^{n}+\int_{t_{0}^{n}}^{t_{1}^{n}} f_{0}^{n}(s) d s\right) \\
\leq & \int_{t_{0}^{n}}^{t_{1}^{n}}\left(k M+g(s)+p(s)\|\varphi\|_{\infty}+q(s)\|\bar{\varphi}\|_{\infty}\right) d s .
\end{aligned}
$$

On the other hand, by (3.1), we have

$$
\begin{aligned}
& \left\|u_{1}^{n}-\bar{\varphi}(0)\right\| \\
\leq & \left\|u_{1}^{n}-\left(u_{0}^{n}+\int_{t_{0}^{n}}^{t_{1}^{n}} f_{0}^{n}(s) d s\right)\right\|+\int_{t_{0}^{n}}^{t_{1}^{n}}\left\|f_{0}^{n}(s)\right\| d s \\
\leq & \int_{t_{0}^{n}}^{t_{1}^{n}}\left(k M+2 g(s)+2 p(s)\|\varphi\|_{\infty}+2 q(s)\|\bar{\varphi}\|_{\infty}\right) d s \\
\leq & \frac{r}{2} .
\end{aligned}
$$


Then $u_{1}^{n} \in \bar{B}(\bar{\varphi}(0), r)$. Now, set

$$
u_{n}(t)=u_{0}^{n}+\frac{\alpha(t)-\alpha\left(t_{0}^{n}\right)}{\alpha\left(t_{1}^{n}\right)-\alpha\left(t_{0}^{n}\right)}\left(u_{1}^{n}-u_{0}^{n}-\int_{t_{0}^{n}}^{t_{1}^{n}} f_{0}^{n}(s) d s\right)+\int_{t_{0}^{n}}^{t} f_{0}^{n}(s) d s
$$

for all $t \in\left[t_{0}^{n}, t_{1}^{n}\right]$, where

$$
\alpha(t)=\int_{0}^{t}(k M+\delta(s)) d s, \forall t \in[0, T] .
$$

So for all $t \in\left[t_{0}^{n}, t_{1}^{n}\right]$

$$
\begin{aligned}
& \left\|u_{n}(t)-\bar{\varphi}(0)\right\| \\
& \leq \frac{\alpha(t)-\alpha\left(t_{0}^{n}\right)}{\alpha\left(t_{1}^{n}\right)-\alpha\left(t_{0}^{n}\right)}\left\|u_{1}^{n}-u_{0}^{n}-\int_{t_{0}^{n}}^{t_{1}^{n}} f_{0}^{n}(s) d s\right\|+\int_{t_{0}^{n}}^{t}\left\|f_{0}^{n}(s)\right\| d s \\
& \leq \alpha(t)-\alpha\left(t_{0}^{n}\right)+\int_{t_{0}^{n}}^{t} \delta(s) d s \\
& \leq \int_{t_{0}^{n}}^{t}(k M+2 \delta(s)) d s \\
& \leq \frac{r}{2}
\end{aligned}
$$

and

$$
\left\|x_{n}(t)-\varphi(0)\right\|=\left\|\int_{t_{0}^{n}}^{t} u_{0}^{n} d s\right\| \leq \int_{t_{0}^{n}}^{t}\left\|u_{0}^{n}\right\| d s \leq \int_{t_{0}^{n}}^{t} M d s \leq \frac{r}{2}
$$

which equivalent to $u_{n}(t) \in \bar{B}\left(\bar{\varphi}(0), \frac{r}{2}\right)$ and $x_{n}(t) \in \bar{B}\left(\varphi(0), \frac{r}{2}\right)$ for all $t \in$ $\left[t_{0}^{n}, t_{1}^{n}\right]$. Now, we have to estimate $\left\|\left(T\left(t_{1}^{n}\right) x_{n}\right)(s)-\varphi(s)\right\|$ and $\|\left(T\left(t_{1}^{n}\right) u_{n}\right)(s)-$ $\bar{\varphi}(s) \|$ for each $s \in[-a, 0]$. If $-t_{1}^{n} \leq s \leq 0$, then $t_{1}^{n}+s \in\left[t_{0}^{n}, t_{1}^{n}\right]$. Thus, by the fact that $|s| \leq t_{1}^{n} \leq T<\eta\left(\frac{r}{2}\right)$, we have

$$
\begin{aligned}
\left\|\left(T\left(t_{1}^{n}\right) x_{n}\right)(s)-\varphi(s)\right\| & =\left\|x_{n}\left(t_{1}^{n}+s\right)-\varphi(s)\right\| \\
& \leq\left\|x_{n}\left(t_{1}^{n}+s\right)-\varphi(0)\right\|+\|\varphi(s)-\varphi(0)\| \\
& \leq r
\end{aligned}
$$

and

$$
\begin{aligned}
\left\|\left(T\left(t_{1}^{n}\right) u_{n}\right)(s)-\bar{\varphi}(s)\right\| & =\left\|u_{n}\left(t_{1}^{n}+s\right)-\bar{\varphi}(s)\right\| \\
& \leq\left\|u_{n}\left(t_{1}^{n}+s\right)-\bar{\varphi}(0)\right\|+\|\bar{\varphi}(s)-\bar{\varphi}(0)\| \\
& \leq r .
\end{aligned}
$$

If $-a \leq s \leq-t_{1}^{n}$, then $t_{1}^{n}+s \in[-a, 0]$. So

$$
\left\|\left(T\left(t_{1}^{n}\right) x_{n}\right)(s)-\varphi(s)\right\|=\left\|\varphi\left(t_{1}^{n}+s\right)-\varphi(s)\right\| \leq r
$$

and

$$
\left\|\left(T\left(t_{1}^{n}\right) u_{n}\right)(s)-\bar{\varphi}(s)\right\|=\left\|\bar{\varphi}\left(t_{1}^{n}+s\right)-\bar{\varphi}(s)\right\| \leq r .
$$

Therefore, $T\left(t_{1}^{n}\right) x_{n} \in \bar{B}_{a}(\varphi, r)$ and $T\left(t_{1}^{n}\right) u_{n} \in \bar{B}_{a}(\bar{\varphi}, r)$. 
We reiterate this process for constructing sequences $\left(f_{i}^{n}(\cdot)\right)_{i},\left(x_{i}^{n}\right)_{i},\left(u_{i}^{n}\right)_{i}$ satisfying, for all $0 \leq i \leq 2^{n}-1$ and for all $t \in\left[t_{i}^{n}, t_{i+1}^{n}\right]$, the following assertions:

$$
\begin{gathered}
f_{i}^{n}(t) \in F\left(t, T\left(t_{i}^{n}\right) x_{n}, T\left(t_{i}^{n}\right) u_{n}\right) \\
\cap \bar{B}\left(0, g(t)+p(t)\left\|T\left(t_{i}^{n}\right) x_{n}\right\|_{\infty}+q(t)\left\|T\left(t_{i}^{n}\right) u_{n}\right\|_{\infty}\right), \\
u_{0}^{n} \in C\left(x_{n}\left(t_{0}^{n}\right)\right), u_{i+1}^{n} \in C\left(x_{n}\left(t_{i+1}^{n}\right)\right) \cap \bar{B}(\bar{\varphi}(0), r), \\
x_{n}(t) \in \bar{B}\left(\varphi(0), \frac{r}{2}\right), T\left(t_{i}^{n}\right) x_{n} \in \bar{B}_{a}(\varphi, r), \\
u_{n}(t) \in \bar{B}\left(\bar{\varphi}(0), \frac{r}{2}\right), T\left(t_{i}^{n}\right) u_{n} \in \bar{B}_{a}(\bar{\varphi}, r), \\
\left\|f_{i}^{n}(t)-y(t)\right\| \leq d\left(y(t), F\left(t, T\left(t_{i}^{n}\right) x_{n}, T\left(t_{i}^{n}\right) u_{n}\right)\right)+\frac{1}{n},
\end{gathered}
$$

$$
\left\|u_{i+1}^{n}-\left(u_{i}^{n}+\int_{t_{i}^{n}}^{t_{i+1}^{n}} f_{i}^{n}(s) d s\right)\right\| \leq \int_{t_{i}^{n}}^{t_{i+1}^{n}}(k M+\delta(s)) d s
$$

$$
\begin{gathered}
u_{n}(t)=u_{i}^{n}+\frac{\alpha(t)-\alpha\left(t_{i}^{n}\right)}{\alpha\left(t_{i+1}^{n}\right)-\alpha\left(t_{i}^{n}\right)}\left(u_{i+1}^{n}-u_{i}^{n}-\int_{t_{i}^{n}}^{t_{i+1}^{n}} f_{i}^{n}(s) d s\right)+\int_{t_{i}^{n}}^{t} f_{i}^{n}(s) d s . \\
x_{n}(t)=x_{i}^{n}+\left(t-t_{i}^{n}\right) u_{i}^{n} .
\end{gathered}
$$

Now, we define the functions $\theta_{n}(\cdot), \bar{\theta}_{n}(\cdot):[0, T] \rightarrow[0, T]$ and $f_{n}(\cdot) \in$ $L^{1}([0, T], H)$ by setting for all $t \in\left[t_{i}^{n}, t_{i+1}^{n}[\right.$

$$
\theta_{n}(t)=t_{i}^{n}, \theta_{n}(T)=T, f_{n}(t)=f_{i}^{n}(t), f_{n}(T)=f_{2^{n}-1}^{n}(T),
$$

and for all $\left.t \in] t_{i}^{n}, t_{i+1}^{n}\right]$

$$
\bar{\theta}_{n}(t)=t_{i+1}^{n}, \bar{\theta}_{n}(0)=0 .
$$

At this stage, the assertions (a)-(b) in Lemma 3.1 are satisfied. Next, we claim that $x_{n}(\cdot)$ and $u_{n}(\cdot)$ are absolutely continuous. Indeed, for all $0 \leq i \leq 2^{n}-1$ and for all $t$ and $s$ in $\left[t_{i}^{n}, t_{i+1}^{n}\right], s<t$, one has

$$
x_{n}(t)-x_{n}(s)=(t-s) u_{i}^{n}
$$

and

$u_{n}(t)-u_{n}(s)=\frac{\alpha(t)-\alpha(s)}{\alpha\left(t_{i+1}^{n}\right)-\alpha\left(t_{i}^{n}\right)}\left(u_{i+1}^{n}-u_{i}^{n}-\int_{t_{i}^{n}}^{t_{i+1}^{n}} f_{i}^{n}(s) d s\right)+\int_{s}^{t} f_{i}^{n}(\tau) d \tau$.

Then, by (3.5) we get

$$
\left\|x_{n}(t)-x_{n}(s)\right\| \leq(t-s) M
$$


and

$$
\begin{aligned}
& \left\|u_{n}(t)-u_{n}(s)\right\| \\
= & \frac{\alpha(t)-\alpha(s)}{\alpha\left(t_{i+1}^{n}\right)-\alpha\left(t_{i}^{n}\right)}\left\|u_{i+1}^{n}-u_{i}^{n}-\int_{t_{i}^{n}}^{t_{i+1}^{n}} f_{i}^{n}(s) d s\right\|+\int_{s}^{t} \delta(\tau) d \tau \\
\leq & \int_{s}^{t}(k M+2 \delta(\tau)) d \tau .
\end{aligned}
$$

By addition, this last inequalities hold for all $s, t \in[0, T]$ with $s<t$. Hence $x_{n}(\cdot)$ and $u_{n}(\cdot)$ are absolutely continuous. Remark that for all $0 \leq i \leq 2^{n}-1$ and for almost every $t$ in $\left[t_{i}^{n}, t_{i+1}^{n}\right]$,

$$
\dot{u}_{n}(t)=\frac{\dot{\alpha}(t)}{\alpha\left(t_{i+1}^{n}\right)-\alpha\left(t_{i}^{n}\right)}\left(u_{i+1}^{n}-u_{i}^{n}-\int_{t_{i}^{n}}^{t_{i+1}^{n}} f_{i}^{n}(s) d s\right)+f_{n}(t) .
$$

Then, by (3.5) we obtain for almost every $t \in[0, T]$

$$
\left\|\dot{u}_{n}(t)-f_{n}(t)\right\| \leq k M+\delta(t) .
$$

Also by construction and the relation (3.4), we have for almost every $t \in[0, T]$

$$
\left(\dot{u}_{n}(t)-f_{n}(t)\right) \in-N_{C\left(x_{n}\left(\bar{\theta}_{n}(t)\right)\right)}\left(u_{n}\left(\bar{\theta}_{n}(t)\right)\right) .
$$

Then the proof is complete.

Proof of Theorem 2.6. In view of Lemma 3.1, we can define inductively sequences $\left(f_{n}(\cdot)\right)_{n \geq 1} \subset L^{1}([0, T], H),\left(x_{n}(\cdot)\right)_{n \geq 1},\left(u_{n}(\cdot)\right)_{n \geq 1} \subset \mathcal{C}([-a, T], H)$ and $\left(\theta_{n}(\cdot)\right)_{n \geq 1},\left(\bar{\theta}_{n}(\cdot)\right)_{n \geq 1} \subset S([0, T],[0, T])$; where $S([0, T],[0, T])$ denotes the space of step functions from $[0, T]$ into $[0, T]$; such that

(1) $f_{n}(t) \in F\left(t, T\left(\theta_{n}(t)\right) x_{n}, T\left(\theta_{n}(t)\right) u_{n}\right) \cap \bar{B}\left(0, g(t)+p(t)\left\|T\left(\theta_{n}(t)\right) x_{n}\right\|_{\infty}+\right.$ $\left.q(t)\left\|T\left(\theta_{n}(t)\right) u_{n}\right\|_{\infty}\right), u_{n}\left(\bar{\theta}_{n}(t)\right) \in C\left(x_{n}\left(\bar{\theta}_{n}(t)\right)\right)$ for all $t \in[0, T]$;

(2) $\left\|f_{n+1}(t)-f_{n}(t)\right\| \leq d\left(f_{n}(t), F\left(t, T\left(\theta_{n+1}(t)\right) x_{n+1}, T\left(\theta_{n+1}(t)\right) u_{n+1}\right)\right)+$ $\frac{1}{n+1}$ for all $t \in[0, T]$;

(3) $\left(\dot{u}_{n}(t)-f_{n}(t)\right) \in-N_{C\left(x_{n}\left(\bar{\theta}_{n}(t)\right)\right)}\left(u_{n}\left(\bar{\theta}_{n}(t)\right)\right)$ for almost all $t \in[0, T]$;

(4) $\left\|\dot{u}_{n}(t)-f_{n}(t)\right\| \leq k M+\delta(t)$ for almost every $t \in[0, T]$.

Firstly, note that

$$
x_{n}(t)=x_{0}+\int_{0}^{t} \bar{u}_{n}(s) d s, \quad \forall t \in[0, T],
$$

where $\bar{u}_{n}(t)=u_{i}^{n} \in L$ for all $t \in\left[t_{i}^{n}, t_{i+1}^{n}\left[\right.\right.$ and for all $0 \leq i \leq 2^{n}-1$. Then for all $t \in[0, T], x_{n}(t) \in x_{0}+[0, T] L$ which is compact. Since $\left\|\dot{x}_{n}(t)\right\| \leq M$, for almost every $t \in[0, T]$, by Arzela-Ascoli's Theorem (see [2]), we can select a subsequence, again denoted by $\left(x_{n}(\cdot)\right)_{n}$ which converges uniformly to an absolutely continuous function $x(\cdot)$ on $[0, T]$, moreover $\dot{x}_{n}(\cdot)$ converges weakly to $\dot{x}(\cdot)$ in $L^{1}([0, T], H)$. Also, since all functions $x_{n}(\cdot)$ agree with $\varphi(\cdot)$ on $[-a, 0]$, we can obviously say that $x_{n}(\cdot)$ converges uniformly to $x(\cdot)$ on $[-a, T]$, if we 
extend $x(\cdot)$ in such a way that $x(\cdot) \equiv \varphi(\cdot)$ on $[-a, 0]$. Secondly, from (4) we deduce

$$
\left\|\dot{u}_{n}(t)\right\| \leq k M+2 \delta(t)
$$

for almost every $t \in[0, T]$. In order to apply Arzela-Ascoli's Theorem we are going to show that for every $t \in[0, T]$ the set $Z(t)=\left\{u_{n}(t): n \geq 1\right\}$ is relatively compact in $H$. By construction we have for all $n \geq 1$ and all $t \in[0, T]$, $u_{n}\left(\bar{\theta}_{n}(t)\right) \in C\left(x_{n}\left(\bar{\theta}_{n}(t)\right)\right) \subset L$. Thus for all $t \in[0, T]$ the set $\left\{u_{n}\left(\bar{\theta}_{n}(t)\right): n \geq 1\right\}$ is relatively compact in $H$. Now, for all $t \in[0, T]$

$$
\begin{aligned}
\beta(Z(t)) & =\beta\left\{u_{n}(t): n \geq 1\right\} \\
& =\beta\left\{u_{n}(t)-u_{n}\left(\bar{\theta}_{n}(t)\right)+u_{n}\left(\bar{\theta}_{n}(t)\right): n \geq 1\right\} .
\end{aligned}
$$

From (iv) of Lemma 2.5 we get

$$
\beta(Z(t)) \leq \beta\left\{u_{n}(t)-u_{n}\left(\bar{\theta}_{n}(t)\right): n \geq 1\right\}+\beta\left\{u_{n}\left(\bar{\theta}_{n}(t)\right): n \geq 1\right\} .
$$

Since the set $\left\{u_{n}\left(\bar{\theta}_{n}(t)\right): n \geq 1\right\}$ is relatively compact in $H$, by (i) of Lemma $2.5, \beta\left\{u_{n}\left(\bar{\theta}_{n}(t)\right): n \geq 1\right\}=0$. Then

$$
\begin{aligned}
\beta(Z(t)) & \leq \beta\left\{u_{n}(t)-u_{n}\left(\bar{\theta}_{n}(t)\right): n \geq 1\right\} \\
& \leq \beta\left\{\int_{t}^{\bar{\theta}_{n}(t)} \dot{u}_{n}(s) d s: n \geq 1\right\} .
\end{aligned}
$$

By relation (3.9) and (v) of Lemma 2.5 we obtain

$$
\begin{aligned}
\beta(Z(t)) & \leq \beta\left\{B\left(0, \int_{t}^{\bar{\theta}_{n}(t)}(k M+2 \delta(s)) d s\right)\right\} \\
& =2 \int_{t}^{\bar{\theta}_{n}(t)}(k M+2 \delta(s)) d s .
\end{aligned}
$$

Since the right term of the above inequality converges to 0 as $n \rightarrow \infty, \beta(Z(t))=$ 0 . Hence $Z(t)$ is relatively compact in $H$. By Arzela-Ascoli's Theorem, we can select a subsequence, again denoted by $\left(u_{n}(\cdot)\right)_{n}$ which converges uniformly to an absolutely continuous function $u(\cdot)$ on $[0, T]$, moreover $\dot{u}_{n}(\cdot)$ converges weakly to $\dot{u}(\cdot)$ in $L^{1}([0, T], H)$. Also, since all functions $u_{n}(\cdot)$ agree with $\bar{\varphi}(\cdot)$ on $[-a, 0]$, we can say, as above, that $u_{n}(\cdot)$ converges uniformly to $u(\cdot)$ on $[-a, T]$. Additionally, observe that $x_{n}\left(\bar{\theta}_{n}(\cdot)\right)$ converges uniformly to $x(\cdot)$ and $u_{n}\left(\bar{\theta}_{n}(\cdot)\right)$ converges uniformly to $u(\cdot)$ on $[0, T]$. Indeed, by (3.6) and (3.7) for all $t \in[0, T]$, we have

$$
\begin{aligned}
\left\|x_{n}\left(\bar{\theta}_{n}(t)\right)-x(t)\right\| & \leq\left\|x_{n}\left(\bar{\theta}_{n}(t)\right)-x_{n}(t)\right\|+\left\|x_{n}(t)-x(t)\right\| \\
& \leq\left(t-\bar{\theta}_{n}(t)\right) M+\left\|x_{n}(t)-x(t)\right\|
\end{aligned}
$$


and

$$
\begin{aligned}
\left\|u_{n}\left(\bar{\theta}_{n}(t)\right)-u(t)\right\| & \leq\left\|u_{n}\left(\bar{\theta}_{n}(t)\right)-u_{n}(t)\right\|+\left\|u_{n}(t)-u(t)\right\| \\
& \leq \int_{t}^{\bar{\theta}_{n}(t)}(k M+2 \delta(s)) d s+\left\|u_{n}(t)-u(t)\right\| .
\end{aligned}
$$

The right term of the above inequalities converge to 0 , it follows that $x_{n}\left(\bar{\theta}_{n}(\cdot)\right)$ converges uniformly to $x(\cdot)$ and $u_{n}\left(\bar{\theta}_{n}(\cdot)\right)$ converges uniformly to $u(\cdot)$ on $[0, T]$. Therefore, for all $t \in[0, T]$, there exists $0 \leq i \leq 2^{n}-1$ such that $t \in\left[t_{i}^{n}, t_{i+1}^{n}\right]$. By (H1) and (3.6), we have

$$
\begin{aligned}
d\left(u_{n}(t), C(x(t))\right) & \leq\left\|u_{n}(t)-u_{n}\left(t_{i}^{n}\right)\right\|+d\left(u_{n}\left(t_{i}^{n}\right), C(x(t))\right) \\
& \leq\left\|u_{n}(t)-u_{n}\left(t_{i}^{n}\right)\right\|+H\left(C\left(x_{n}\left(t_{i}^{n}\right)\right), C(x(t))\right) \\
& \leq \int_{t_{i}^{n}}^{t}(k M+2 \delta(\tau)) d \tau+k\left\|x_{n}\left(t_{i}^{n}\right)-x(t)\right\| .
\end{aligned}
$$

The right term of the above inequality converges to 0 if $n \rightarrow+\infty$. Then we conclude that $u(t) \in C(x(t))$ for all $t \in[0, T]$.

Claim 3.2. $\dot{x}(t)=u(t)$ for almost all $t \in[0, T]$.

Proof. Let $t \in[0, T]$ such that $\dot{x}_{n}(t)$ and $\dot{x}(t)$ exist. There exists $0 \leq i \leq 2^{n}-1$ such that $t \in\left[t_{i}^{n}, t_{i+1}^{n}\right]$. By construction we have

$$
\begin{aligned}
& \left\|\dot{x}_{n}(t)-u_{n}(t)\right\| \\
\leq & \left\|\frac{\alpha(t)-\alpha\left(t_{i}^{n}\right)}{\alpha\left(t_{i+1}^{n}\right)-\alpha\left(t_{i}^{n}\right)}\left(u_{i+1}^{n}-u_{i}^{n}-\int_{t_{i}^{n}}^{t_{i+1}^{n}} f_{i}^{n}(s) d s\right)\right\|+\left\|\int_{t_{i}^{n}}^{t} f_{i}^{n}(s) d s\right\| \\
\leq & \alpha(t)-\alpha\left(t_{i}^{n}\right)++\int_{t_{i}^{n}}^{t} \delta(s) d s \\
\leq & \int_{t_{i}^{n}}^{t}(k M+2 \delta(s)) d s .
\end{aligned}
$$

Let $\varepsilon>0$. Since $\frac{T}{2^{n}}$ converges to 0 , there exists $n_{0} \in \mathbb{N}$ such that for all $n \geq n_{0}$, $\left|t_{i}^{n}-t\right| \leq \frac{T}{2^{n}}<\eta\left(\frac{\varepsilon}{2}\right)$. Hence by $(3.2)$

$$
\left\|\dot{x}_{n}(t)-u_{n}(t)\right\| \leq \varepsilon, \quad \forall n \geq n_{0} .
$$

Now, since

$$
\left\|\dot{x}_{n}(t)-u(t)\right\| \leq\left\|\dot{x}_{n}(t)-u_{n}(t)\right\|+\left\|u_{n}(t)-u(t)\right\|,
$$

we deduce that $\dot{x}_{n}(\cdot)$ converges uniformly to $u(\cdot)$, so $\dot{x}(t)=u(t)$ and $\ddot{x}(t)=\dot{u}(t)$ for almost all $t \in[0, T]$.

Claim 3.3. $T\left(\theta_{n}(t)\right) x_{n}$ converges to $T(t) x$ and $T\left(\theta_{n}(t)\right) u_{n}$ converges to $T(t) u$ in $\mathcal{C}_{a}$. 
Proof. Let us denote the modulus continuity of a function $\psi(\cdot)$ defined on interval $I$ of $\mathbb{R}$ by

$$
\omega(\psi(\cdot), I, \eta):=\sup \{\|\psi(t)-\psi(s)\|: s, t \in I,|s-t|<\eta\} .
$$

Let $\varepsilon>0$ and let $t, t^{\prime} \in[0, T]$, assume that $0 \leq t^{\prime}-t<\eta\left(\frac{\varepsilon}{2}\right)$. By (3.2), (3.6) and (3.7), we have

$$
\left\|x_{n}(t)-x_{n}\left(t^{\prime}\right)\right\| \leq\left(t^{\prime}-t\right) M \leq \frac{\varepsilon}{2}
$$

and

Hence

$$
\left\|u_{n}(t)-u_{n}\left(t^{\prime}\right)\right\| \leq \int_{t}^{t^{\prime}}(k M+2 \delta(s)) d s \leq \frac{\varepsilon}{2} .
$$

$$
\omega\left(x_{n}(\cdot),[0, T], \eta\left(\frac{\varepsilon}{2}\right)\right) \leq \frac{\varepsilon}{2} \text { and } \omega\left(u_{n}(\cdot),[0, T], \eta\left(\frac{\varepsilon}{2}\right)\right) \leq \frac{\varepsilon}{2} .
$$

Also for $t, t^{\prime} \in[-a, 0]$ such that $\left|t^{\prime}-t\right|<\eta\left(\frac{\varepsilon}{2}\right)$, by (3.2) we have

$$
\left\|\varphi(t)-\varphi\left(t^{\prime}\right)\right\|<\frac{\varepsilon}{2} \text { and }\left\|\bar{\varphi}(t)-\bar{\varphi}\left(t^{\prime}\right)\right\|<\frac{\varepsilon}{2} .
$$

Then

$$
\omega\left(\varphi(\cdot),[-a, 0], \eta\left(\frac{\varepsilon}{2}\right)\right) \leq \frac{\varepsilon}{2} \text { and } \omega\left(\bar{\varphi}(\cdot),[-a, 0], \eta\left(\frac{\varepsilon}{2}\right)\right) \leq \frac{\varepsilon}{2} .
$$

Now, let $t \in[0, T]$, since $\theta_{n}(t)$ converges to $t$, there exists $n_{0} \in \mathbb{N}$ such that for all $n \geq n_{0},\left|\theta_{n}(t)-t\right|<\eta\left(\frac{\varepsilon}{2}\right)$. Then, for all $n \geq n_{0}$

$$
\begin{aligned}
& \left\|T\left(\theta_{n}(t)\right) x_{n}-T(t) x_{n}\right\|_{\infty} \\
= & \sup _{-a \leq s \leq 0}\left\|x_{n}\left(\theta_{n}(t)+s\right)-x_{n}(t+s)\right\| \\
\leq & \omega\left(x_{n}(\cdot),[-a, T], \eta\left(\frac{\varepsilon}{2}\right)\right) \\
\leq & \omega\left(\varphi(\cdot),[-a, 0], \eta\left(\frac{\varepsilon}{2}\right)\right)+\omega\left(x_{n}(\cdot),[0, T], \eta\left(\frac{\varepsilon}{2}\right)\right) \\
\leq & \varepsilon
\end{aligned}
$$

and

$$
\begin{aligned}
& \left\|T\left(\theta_{n}(t)\right) u_{n}-T(t) u_{n}\right\|_{\infty} \\
= & \sup _{-a \leq s \leq 0}\left\|u_{n}\left(\theta_{n}(t)+s\right)-u_{n}(t+s)\right\| \\
\leq & \omega\left(u_{n}(\cdot),[-a, T], \eta\left(\frac{\varepsilon}{2}\right)\right) \\
\leq & \omega\left(\varphi(\cdot),[-a, 0], \eta\left(\frac{\varepsilon}{2}\right)\right)+\omega\left(u_{n}(\cdot),[0, T], \eta\left(\frac{\varepsilon}{2}\right)\right) \\
\leq & \varepsilon,
\end{aligned}
$$

hence $\left\|T\left(\theta_{n}(t)\right) x_{n}-T(t) x_{n}\right\|_{\infty}$ and $\left\|T\left(\theta_{n}(t)\right) u_{n}-T(t) u_{n}\right\|_{\infty}$ converge to 0 as $n \rightarrow+\infty$. Therefore, since the uniform convergence of $x_{n}(\cdot)$ to $x(\cdot)$ and the uniform convergence of $u_{n}(\cdot)$ to $u(\cdot)$ on $[-a, T]$ imply that $T(t) x_{n}$ converges 
to $T(t) x$ and $T(t) u_{n}$ converges to $T(t) u$ uniformly on $[-a, 0]$, we deduce that $T\left(\theta_{n}(t)\right) x_{n}$ converges to $T(t) x$ and $T\left(\theta_{n}(t)\right) u_{n}$ converges to $T(t) u$ in $\mathcal{C}_{a}$.

Porposition 3.4. $x(\cdot)$ is a solution of $(2.1)$.

Proof. Let $t \in[0, T]$, from (1) and (2) we deduce

$$
\begin{aligned}
& \left\|f_{n+1}(t)-f_{n}(t)\right\| \\
\leq & H\left(F\left(t, T\left(\theta_{n}(t)\right) x_{n}, T\left(\theta_{n}(t)\right) u_{n}\right), F\left(t, T\left(\theta_{n+1}(t)\right) x_{n+1},\right.\right. \\
& \left.\left.T\left(\theta_{n+1}(t)\right) u_{n+1}\right)\right)+\frac{1}{n+1} \\
\leq & m(t)\left\|T\left(\theta_{n}(t)\right) u_{n}-T\left(\theta_{n+1}(t)\right) u_{n+1}\right\|_{\infty}+\frac{1}{n+1} .
\end{aligned}
$$

By Claim 3.3, $\left\|T\left(\theta_{n}(t)\right) u_{n}-T\left(\theta_{n+1}(t)\right) u_{n+1}\right\|_{\infty}$ converges to 0 , then the right term of the relation (3.10) converges to 0 . Hence $\left(f_{n}(t)\right)_{n \geq 1}$ is a Cauchy sequence and $f_{n}(t)$ converges to $f(t)$. Moreover, observe that by $(1)$,

$$
\begin{aligned}
& d(f(t), F(t, T(t) x, T(t) u)) \\
\leq & \left\|f(t)-f_{n}(t)\right\|+H\left(F\left(t, T\left(\theta_{n}(t)\right) x_{n}, T\left(\theta_{n}(t)\right) u_{n}\right), F(t, T(t) x, T(t) u)\right) \\
\leq & \left\|f(t)-f_{n}(t)\right\|+m(t)\left\|T\left(\theta_{n}(t)\right) u_{n}-T(t) u\right\|_{\infty} .
\end{aligned}
$$

Since $f_{n}(t)$ converges to $f(t)$ and by Claim 3.3 , the last term converges to 0 . So that $f(t) \in F(t, T(t) x, T(t) u)$ for all $t \in[0, T]$. Now, the weak convergence of $\dot{u}_{n}(\cdot)$ to $\dot{u}(\cdot)$ in $L^{1}([0, T], H)$ and the Mazur's Lemma entail

$$
\dot{u}(t)-f(t) \in \bigcap_{n} \overline{c o}\left\{\dot{u}_{m}(t)-f_{m}(t): m \geq n\right\} \text { for a.e. on }[0, T] \text {. }
$$

Fix any $t \in[0, T]$ and $y \in H$, we have

$$
\langle y, \dot{u}(t)-f(t)\rangle \leq \inf _{n} \sup _{k \geq n}\left\langle y, \dot{u}_{k}(t)-f_{k}(t)\right\rangle .
$$

By (3) and (4), one has

$$
\left(\dot{u}_{n}(t)-f_{n}(t)\right) \in-N_{C\left(x_{n}\left(\bar{\theta}_{n}(t)\right)\right)}\left(u_{n}\left(\bar{\theta}_{n}(t)\right)\right) \cap \gamma(t) \bar{B},
$$

where $\gamma(t)=k M+\delta(t)$. Hence, by Proposition 2.1 we get

$$
\left(\dot{u}_{n}(t)-f_{n}(t)\right) \in-\gamma(t) \partial d_{C\left(x_{n}\left(\bar{\theta}_{n}(t)\right)\right)}\left(u_{n}\left(\bar{\theta}_{n}(t)\right)\right) .
$$

In view of Proposition 2.2, we deduce

$$
\begin{aligned}
& \langle y, \dot{u}(t)-f(t)\rangle \\
\leq & \gamma(t) \limsup _{n \rightarrow \infty} \sigma\left(y,-\partial d_{C\left(x_{n}\left(\bar{\theta}_{n}(t)\right)\right)}\left(u_{n}\left(\bar{\theta}_{n}(t)\right)\right)\right) \\
\leq & \gamma(t) \sigma\left(y,-\partial d_{C(x(t))}(u(t))\right) .
\end{aligned}
$$

So, the convexity and the closedness of the set $\partial d_{C(x(t))}(u(t))$ ensure

$$
(\dot{u}(t)-f(t)) \in-\gamma(t) \partial d_{C(x(t))}(u(t)) \subset-N_{C(x(t))}(u(t)) .
$$


Finally, we have

$$
\dot{u}(t) \in-N_{C(x(t))}(u(t))+F(t, T(t) x, T(t) u)
$$

and $u(t) \in C(x(t))$. By Claim 3.2, we get

$$
\ddot{x}(t) \in-N_{C(x(t))}(\dot{x}(t))+F(t, T(t) x, T(t) \dot{x})
$$

and $\dot{x}(t) \in C(x(t))$. The proof is complete.

Acknowledgements. The author would like to thank the referee for his careful and thorough reading of the paper.

\section{References}

[1] R. P. Agarwal and D. Oregan, A survey of recent fixed point theory in Frechet spaces, Nonlinear analysis and applications: to V. Lakshmikantham on his 80th birthday. Vol. 1, 2, 75-88, Kluwer Acad. Publ., Dordrecht, 2003,

[2] J. P. Aubin and A. Cellina, Differential Inclusions, Springer-Verlag, Berlin Heidelberg, 1984.

[3] D. Azzam-Laouir, Mixed semicontinuous perturbation of a second order nonconvex sweeping process, Electron. J. Qual. Theory Differ. Equ. 2008 (2008), no. 37, 1-9.

[4] H. Benabdellah, C. Castaing, A. Salvadori, and A. Syam, Nonconvex sweeping process, J. Appl. Anal. 2 (1996), no. 2, 217-240.

[5] M. Bounkhel, General existence results for second order nonconvex sweeping process with unbounded perturbations, Port. Math. (N.S.) 60 (2003), no. 3, 269-304.

[6] M. Bounkhel and D. Laouir-Azzam, Existence results on the second-order nonconvex sweeping process with perturbations, Set-Valued Var. Anal. 12 (2004), no. 3, 291-318.

[7] M. Bounkhel and L. Thibault, Nonconvex sweeping process and prox-regularity in Hilbert space, J. Nonlinear Convex Anal. 6 (2005), no. 2, 359-374.

[8] M. Bounkhel and M. Yarou, Existence results for first and second order nonconvex sweeping process with delay, Port. Math. (N.S.) 61 (2004), no. 2, 207-230.

[9] C. Castaing, Quelques problemes d'evolution du second ordre, Seminaire d'Analyse Convexe, Vol. 18 (Montpellier, 1988), Exp. No. 5, 18 pp., Univ. Sci. Tech. Languedoc, Montpellier, 1988 ,

[10] C. Castaing and M. D. P. Monteiro Marques, Topological properties of solution sets for sweeping process with delay, Port. Math. 54 (1997), no. 4, 485-507.

[11] C. Castaing and M. Valadier, Convex Analysis and Measurable Multifunctions, Lecture Notes in Mathematics, Vol. 580, Springer-Verlag, Berlin-Heidelberg-New York, 1977.

[12] C. Castaing, M. Valadier, and T. X. Ducha, Evolution equations governed by the sweeping process, Set-Valued Var. Anal. 1 (1993), no. 2, 109-139.

[13] F. H. Clarke, R. J. Stern, and P. R. Wolenski, Proximal smoothness and the lower $C^{2}$ property, J. Convex Anal. 2 (1995), no. 1-2, 117-144.

[14] K. Deimling, Multivalued Differential Equations, De Gruyter Series in Non linear Analysis and Applications, Walter de Gruyter, Berlin, New York, 1992.

[15] T. X. Duc Ha and M. D. P. Monteiro-Marques, Nonconvex second-order differential inclusions with memory, Set-Valued Var. Anal. 3 (1995), no. 1, 71-86.

[16] J. F. Edmond, Delay perturbed sweeping process, Set-Valued Var. Anal. 14 (2006), no. $3,295-317$.

[17] T. Haddad and L. Thibault, Mixed semicontinuous perturbations of nonconvex sweeping process, Math. Program. 123 (2010), no. 1, Ser. B, 225-240.

[18] M. D. P. Monteiro Marques, Differential inclusions in nonsmooth mechanical problems, Shocks and dry friction. Progress in Nonlinear Differential Equations and their Applications, 9. Birkhauser Verlag, Basel, 1993. 
[19] J. J. Moreau, Evolution problem associated with a moving convex set in a Hilbert space, J. Differential Equations 26 (1977), no. 3, 347-374.

[20] _ Application of convex analysis to the treatment of elasto-plastic systems, in "Applications of Methods of Functional Analysis to Problems in Mechanics", (Germain and Nayroles, Eds.), Lecture Notes in Mathematics, 503, Springer-Verlag, Berlin, (1976), $56-89$.

[21] _ Unilateral contact and dry friction in finite freedom dynamics, in "Nonsmooth Mechanics", (J.J. Moreau and P.D. Panagiotopoulos, Eds.), CISM Courses and Lectures, 302, Springer-Verlag, Vienna, New York, (1988), 1-82.

[22] R. A. Poliquin, R. T. Rockafellar, and L. Thibault, Local differentiability of distance functions, Trans. Amer. Math. Soc. 352 (2000), no. 11, 5231-5249.

[23] Q. Zhu, On the solution set of differential inclusions in Banach space, J. Differential Equations 93 (1991), no. 2, 213-237.

High SChOOL IBN Khaldoune

COMMUNE BOUZNIKA

BP 13100, Morocco

E-mail address: aitalifr@hotmail.com 